CRYSTALLOGRAPHIC COMMUNICATIONS

ISSN 2056-9890

Received 18 August 2017

Accepted 22 August 2017

Edited by M. Zeller, Purdue University, USA

Keywords: crystal structure; cage compounds; nitrogen heterocycles; amine; alkaloid; epibatidine.

CCDC reference: 1511233

Supporting information: this article has supporting information at journals.iucr.org/e
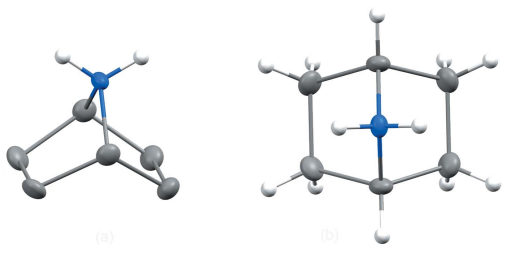

OPEN $\odot$ ACCESS

\section{The 7-azanorbornane nucleus of epibatidine: 7-azabicyclo[2.2.1]heptan-7-ium chloride}

\author{
Sergey N. Britvin ${ }^{\mathrm{a} *}$ and Andrey M. Rumyantsev ${ }^{\mathrm{b}}$
}

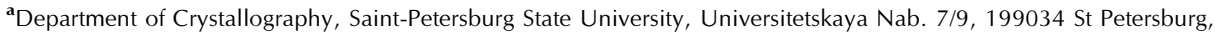
Russian Federation, and ${ }^{\mathbf{b}}$ Department of Genetics and Biotechnology, Saint-Petersburg State University, Universitetskaya Nab. 7/9, 199034 St Petersburg, Russian Federation. *Correspondence e-mail: sergei.britvin@spbu.ru

7-Azabicyclo[2.2.1]heptane (7-azanorbornane) is a bridged heterocyclic nucleus found in epibatidine, the alkaloid isolated from the skin of the tropical poison frog Epipedobates tricolor. Since epibatidine is known as one of the most potent acetylcholine nicotinic receptor agonists, a plethora of literature has been devoted to this alkaloid. However, there are no structural data on the unsubstituted 7-azanorbornane, the parent bicyclic ring of epibatidine and its derivatives. We herein present the structural characterization of the 7azabicyclo[2.2.1]heptane parent ring as its hydrochloride salt, namely 7azabicyclo[2.2.1] heptan-7-ium chloride, $\mathrm{C}_{6} \mathrm{H}_{12} \mathrm{~N}^{+} \cdot \mathrm{Cl}^{-}$. The compete cation is generated by a crystallographic mirror plane with the $\mathrm{N}$ atom lying on the mirror, as does the chloride anion. In the crystal, the cations are linked to the anions by $\mathrm{N}-\mathrm{H} \cdots \mathrm{Cl}$ hydrogen bonds, which generate [001] chains.

\section{Chemical context}

Since the discovery of the quinuclidine and tropane nuclei (Hamama et al., 2006; Pollini et al., 2006), elegant frameworks of bridged aza-heterocycles have been the focus of chemists exploring biologically active substances. One famous example in this series is epibatidine, (-)-2-(6-chloropyridin-3-yl)-7azabicyclo[2.2.1]heptane, an active component of the skin poison extracted from the small tropical frog Epipedobates tricolor (Spande et al., 1992; Gerzanich et al., 1995; Sullivan \& Bannon, 1996; Dukat \& Glennon, 2003). Epibatidine comprises the first natural example of a compound incorporating an 7-azabicyclo[2.2.1]heptane (7-azanorbornane) ring system (Fletcher et al., 1994). Due to the extreme binding affinity of the exo isomer of epibatidine towards nicotinic acetylcholine receptors, thousands of articles have been devoted to different aspects of its chemistry and biochemistry (see Carroll, 2004; Daly et al., 2005; Yogeeswari et al., 2006; Garraffo et al., 2009). We are not aware, however, that an $\mathrm{X}$-ray structure determination of the alkaloid itself has ever been reported, in spite of numerous publications related to its

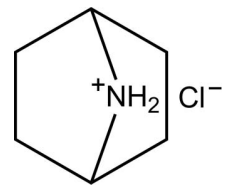

synthesis. Moreover, the molecular structure of 7-azanorbornane, the functional core of epibatidine, has also not been explored, in spite of the fact that 7-azanorbornane has been known since 1930 (Braun \& Schwarz, 1930; Fraser \& Swingle, 1970). In continuation of our studies related to bridged aza-heterocyclic systems (Britvin et al., 2015, 2016, 
Table 1

Hydrogen-bond geometry $\left(\AA,^{\circ}\right)$.

\begin{tabular}{lllll}
\hline$D-\mathrm{H} \cdots A$ & $D-\mathrm{H}$ & $\mathrm{H} \cdots A$ & $D \cdots A$ & $D-\mathrm{H} \cdots A$ \\
\hline $\mathrm{N} 7-\mathrm{H} 7 B \cdots \mathrm{Cl} 1^{\mathrm{i}}$ & $0.88(3)$ & $2.25(3)$ & $3.127(2)$ & $175(2)$ \\
$\mathrm{N} 7-\mathrm{H} 7 A \cdots \mathrm{Cl} 1$ & $0.87(4)$ & $2.25(4)$ & $3.122(2)$ & $178(3)$ \\
\hline
\end{tabular}

Symmetry code: (i) $-x+1,-y+1, z+\frac{1}{2}$.

2017), we herein report on the structure of the unsubstituted 7-azabicyclo[2.2.1]heptane parent ring as its hydrochloride salt, namely 7-azabicyclo[2.2.1] heptan-7-ium chloride, $\mathbf{1}$.

\section{Structural commentary}

The parent ring of 7-azabicyclo[2.2.1] heptane in $\mathbf{1}$ adopts a boat conformation (Fig. 1) resembling the molecular geometry of its nearest carbocyclic counterpart, bicyclo[2.2.1] heptane (norbornane), 2 (Fitch \& Jobic, 1993). In order to achieve consistency of atomic labelling between the bicyclic cages of $\mathbf{1}$ and $\mathbf{2}$, we herein apply the numbering scheme according to IUPAC nomenclature (Fig. 1) (Doms et al., 1985). There are three unique $\mathrm{C}$ atoms $(\mathrm{C} 1, \mathrm{C} 2$ and $\mathrm{C} 6)$ in the cation of $\mathbf{1}$, with their clones $\mathrm{C}^{\mathrm{i}}{ }^{\mathrm{i}}=\mathrm{C} 4$ by IUPAC; symmetry code: (i) $1-x, y$, $z$ ], $\mathrm{C} 2^{\mathrm{i}}$ (= $\mathrm{C} 3$ by IUPAC) and $\mathrm{C} 6{ }^{\mathrm{i}}$ (= C5 by IUPAC) generated by the mirror at $x=\frac{1}{2}$. Interatomic distances between the respective framework sites of $\mathbf{1}$ are shorter compared with the corresponding values of $\mathbf{2}$. The distances $(\AA)$ in $\mathbf{1}$ and $\mathbf{2}$ are: $\mathrm{C} 1-\mathrm{C} 2=1.528(2)$ and $1.551(3), \mathrm{C} 1-\mathrm{C} 6=1.523$ (3) and $1.578(1)$, and $\mathrm{C} 1-\mathrm{N} 7(\mathrm{C} 7)=1.508(2)$ and 1.551 (3). The $\mathrm{C} 2{ }^{\mathrm{i}}-\mathrm{C} 2-\mathrm{C} 1-\mathrm{C} 6$ torsion angle determining the boat-like conformation is $109.4(1)^{\circ}$ in $\mathbf{1}$ and $108.7(2)^{\circ}$ in $\mathbf{2}$. The s.u. values for $\mathbf{2}$ were generated using PLATON (Spek, 2009). Further details of the interatomic distances and angles of $\mathbf{1}$ can be found in the supporting information.

\section{Supramolecular features}

The structural integrity of $\mathbf{1}$ is maintained via intermolecular hydrogen bonding between the protonated secondary site N7 and the chloride counter-ion Cl1 (Table 1). Each chloride ion is linked to the two adjacent amine centres via $\mathrm{N}-\mathrm{H} \cdots \mathrm{Cl}$ hydrogen bonds so that the 7-azanorbornane cages are arranged into zigzag chains flattened on (010) and propagating along the $c$-axis direction (Fig. 2). That type of interleaved zigzag packing is known among chloride salts of secondary amines, both for alkyl- and arylamines (Adams et al., 1997; Nancy et al., 2003; Muller et al., 2007) and heterocyclic systems (Gribkov et al., 2006; Wang et al., 2011; Fun et al., 2011).

\section{Database survey}

Of more than 120 structures containing the 7 -azanorbornane ring system in the Cambridge Structural Database (CSD, Version 5.38, latest update May 2017; Groom et al., 2016), 17 entries represent the 7-azabicyclo[2.2.1]heptane parent ring unsubstituted at the carbon sites. All these compounds belong to $\mathrm{N}$-substituted derivatives of 7-azanorbornane (Ohwada $e t$
Table 2

Experimental details.

\begin{tabular}{|c|c|}
\hline \multicolumn{2}{|l|}{ Crystal data } \\
\hline Chemical formula & $\mathrm{C}_{6} \mathrm{H}_{12} \mathrm{~N}^{+} \cdot \mathrm{Cl}^{-}$ \\
\hline$M_{\mathrm{r}}$ & 133.62 \\
\hline Crystal system, space group & Orthorhombic, $C m c 2_{1}$ \\
\hline Temperature $(\mathrm{K})$ & 100 \\
\hline$a, b, c(\AA)$ & $9.1532(6), 8.7029(8), 8.7336(5)$ \\
\hline$V\left(\AA^{3}\right)$ & $695.71(9)$ \\
\hline$Z$ & 4 \\
\hline Radiation type & Mo $K \alpha$ \\
\hline$\mu\left(\mathrm{mm}^{-1}\right)$ & 0.45 \\
\hline Crystal size $(\mathrm{mm})$ & $0.08 \times 0.06 \times 0.04$ \\
\hline \multicolumn{2}{|l|}{ Data collection } \\
\hline Diffractometer & Bruker APEXII CCD \\
\hline Absorption correction & $\begin{array}{l}\text { Multi-scan (SADABS; Sheldrick, } \\
\text { 2015) }\end{array}$ \\
\hline $\begin{array}{l}\text { No. of measured, independent and } \\
\text { observed }[I>2 \sigma(I)] \text { reflections }\end{array}$ & $3239,777,769$ \\
\hline$R_{\mathrm{int}}$ & 0.017 \\
\hline$(\sin \theta / \lambda)_{\max }\left(\AA^{-1}\right)$ & 0.638 \\
\hline \multicolumn{2}{|l|}{ Refinement } \\
\hline$R\left[F^{2}>2 \sigma\left(F^{2}\right)\right], w R\left(F^{2}\right), S$ & $0.017,0.048,1.15$ \\
\hline No. of reflections & 777 \\
\hline No. of parameters & 47 \\
\hline No. of restraints & 1 \\
\hline $\mathrm{H}$-atom treatment & $\begin{array}{l}\mathrm{H} \text { atoms treated by a mixture of } \\
\text { independent and constrained } \\
\text { refinement }\end{array}$ \\
\hline$\Delta \rho_{\max }, \Delta \rho_{\min }\left(\mathrm{e} \AA^{-3}\right)$ & $0.21,-0.12$ \\
\hline Absolute structure & Refined as an inversion twin \\
\hline Absolute structure parameter & $0.19(9)$ \\
\hline
\end{tabular}

Computer programs: APEX2 (Bruker, 2015), SAINT (Bruker, 2015), SHELXT (Sheldrick, 2015a), OLEX2 (Dolomanov et al., 2009), SHELXL2014 (Sheldrick, 2015b), Mercury (Macrae et al., 2008) and publCIF (Westrip, 2010).

al. 1998; Cheng et al. 2002; Otani et al. 2003; Hori et al. 2008; Longobardi et al. 2015).

\section{Synthesis and crystallization}

7-Azabicyclo[2.2.1] heptane hydrochloride, 1, was obtained from Sigma Aldrich. The purity of the substance has been proven by elemental analysis (analysis calculated for

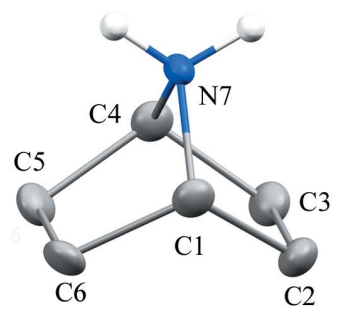

(a)

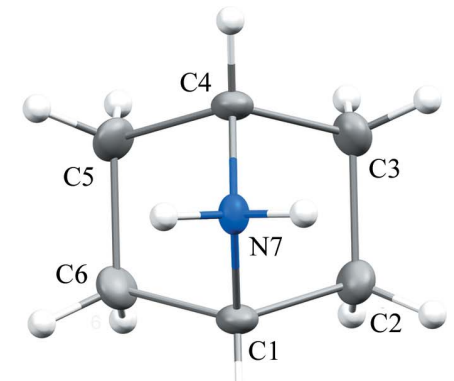

(b)
Figure 1

The molecular structure and systematic atomic numbering scheme of the 7-azabicyclo[2.2.1] heptane (7-azanorbornane) parent ring in 1. Displacement ellipsoids are drawn at the $50 \%$ probability level. $\mathrm{H}$ atoms on $\mathrm{C}$ atoms in view $(a)$ and the chloride counter-ion have been omitted for clarity. The labelling in the Figures corresponds to IUPAC notation (see text). Atoms C4, C3 and C5 are generated from C1, C2 and C6, respectively, by the symmetry operation $(1-x, y, z)$. 


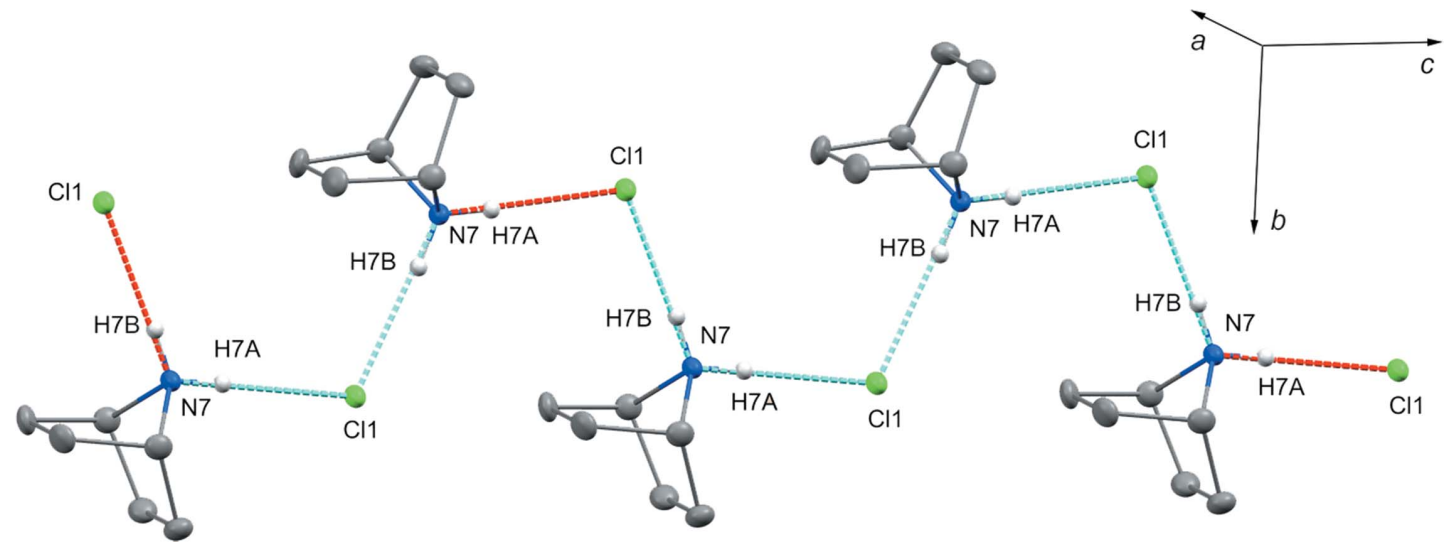

Figure 2

Hydrogen bonding in the crystal structure of $\mathbf{1}$. Protonated molecules of 7 -azanorbornane are linked via $\mathrm{N}-\mathrm{H} \cdots \mathrm{Cl}$ hydrogen bonds to form infinite zigzag chains propagated along the $c$ axis. Displacement ellipsoids are drawn at the 50\% probability level. $\mathrm{H}$ atoms not involved in hydrogen bonding have been omitted for clarity.

$\mathrm{C}_{6} \mathrm{H}_{12} \mathrm{ClN}$ : C 53.93, H 9.05, N 10.48\%; found: C 53.89, H 9.08, $\mathrm{N} 10.44 \%$ ). ${ }^{1} \mathrm{H}$ NMR (400 MHz) spectrum (Bruker Avance 400, $\mathrm{SiMe}_{4}$ external standard, $\mathrm{D}_{2} \mathrm{O}$ solution): $\delta 4.21-4.19(\mathrm{~m}$, $2 \mathrm{H}, 2 \times \mathrm{CH}$ at $\mathrm{C} 1$ and $\mathrm{C} 4$; the atom-numbering scheme is according to IUPAC nomenclature, see Fig. 1), 1.92-1.84 ( $m$, $4 \mathrm{H}, 4 \times$ endo- $\mathrm{HCH}$ at $\mathrm{C} 2, \mathrm{C} 3, \mathrm{C} 5, \mathrm{C} 6), 1.78-1.71(m, 4 \mathrm{H}, 4 \times$ exo-HCH at $\mathrm{C} 2, \mathrm{C} 3, \mathrm{C} 5, \mathrm{C} 6) .{ }^{13} \mathrm{C}\left\{{ }^{1} \mathrm{H}\right\} \mathrm{NMR}(101 \mathrm{MHz}): \delta 58.9$ ( $s, \mathrm{C} 1$ and C4), 26.7 ( $s, \mathrm{C} 2, \mathrm{C} 3, \mathrm{C} 5, \mathrm{C} 6)$. Crystals of 1 suitable for structural studies were obtained by slow evaporation of its aqueous solution.

\section{Refinement}

$\mathrm{H}$ atoms at the protonated $\mathrm{N} 7$ atom were refined freely, whereas $\mathrm{H}$ atoms on $\mathrm{C}$ atoms were refined based on a riding model. Crystal data, data collection and structure refinement details are summarized in Table 2.

\section{Acknowledgements}

The authors thank the X-ray Diffraction Center, Center for Magnetic Resonance and Computer Resource Center of Saint-Petersburg State University for instrumental and computational resources.

\section{Funding information}

Funding for this research was provided by: Saint-Petersburg State University (grant Nos. 0.37.235.2015 and 3.37.222.2015).

\section{References}

Adams, C., Raithby, P. R. \& Davies, J. E. (1997). Private communication (deposition number 100996). CCDC, Cambridge, England.

Braun, J. \& Schwarz, K. (1930). Justus Liebigs Ann. Chem. 481, 56-68. Britvin, S. N. \& Lotnyk, A. (2015). J. Am. Chem. Soc. 137, 5526-5535.

Britvin, S. N., Rumyantsev, A. M., Zobnina, A. E. \& Padkina, M. V. (2016). Chem. Eur. J. pp. 14227-14235.

Britvin, S. N., Rumyantsev, A. M., Zobnina, A. E. \& Padkina, M. V. (2017). J. Mol. Struct. 1130, 395-399.

Bruker (2015). APEX2 and SAINT. Bruker AXS Inc., Madison, Wisconsin, USA.
Carroll, I. F. (2004). Bioorg. Med. Chem. Lett. 14, 1889-1896.

Cheng, J., Zhang, C., Stevens, E. D., Izenwasser, S., Wade, D., Chen, S., Paul, D. \& Trudell, M. L. (2002). J. Med. Chem. 45, 3041-3047.

Daly, J. W., Spande, T. F. \& Garraffo, H. M. (2005). J. Nat. Prod. 68, 1556-1575.

Dolomanov, O. V., Bourhis, L. J., Gildea, R. J., Howard, J. A. K. \& Puschmann, H. (2009). J. Appl. Cryst. 42, 339-341.

Doms, L., Van Hemelrijk, D., Van de Mieroop, W., Lenstra, A. T. H. \& Geise, H. J. (1985). Acta Cryst. B41, 270-274.

Dukat, M. \& Glennon, R. A. (2003). Cell. Mol. Neurobiol. 23, 365378.

Fitch, A. N. \& Jobic, H. (1993). J. Chem. Soc. Chem. Commun. pp. 1516-1517.

Fletcher, S. R., Baker, R., Chambers, M. S., Herbert, R. H., Hobbs, S. C., Thomas, S. R., Verrier, H. M., Watt, A. P. \& Ball, R. G. (1994). J. Org. Chem. 59, 1771-1778.

Fraser, R. R. \& Swingle, R. B. (1970). Can. J. Chem. 48, 2065-2074.

Fun, H.-K., Asik, S. I. J., Chandrakantha, B., Isloor, A. M. \& Shetty, P. (2011). Acta Cryst. E67, o3115.

Garraffo, H. M., Spande, T. F. \& Williams, M. (2009). Heterocycles, 79, 207-217.

Gerzanich, V., Peng, X., Wang, F., Wells, G., Anand, R., Fletcher, S. \& Lindstrom, J. (1995). Mol. Pharmacol. 48, 774-782.

Gribkov, D. V., Hultzsch, K. C. \& Hampel, F. (2006). J. Am. Chem. Soc. 128, 3748-3759.

Groom, C. R., Bruno, I. J., Lightfoot, M. P. \& Ward, S. C. (2016). Acta Cryst. B72, 171-179.

Hamama, W. S., Abd El-Magid, O. M. \& Zoorob, H. H. (2006). Heterocycl. Chem. 43, 1397-1420.

Hori, T., Otani, Y., Kawahata, M., Yamaguchi, K. \& Ohwada, T. (2008). J. Org. Chem. 73, 9102-9108.

Longobardi, L. E., Mahdi, T. \& Stephan, D. W. (2015). Dalton Trans. 44, 7114-7117.

Macrae, C. F., Bruno, I. J., Chisholm, J. A., Edgington, P. R., McCabe, P., Pidcock, E., Rodriguez-Monge, L., Taylor, R., van de Streek, J. \& Wood, P. A. (2008). J. Appl. Cryst. 41, 466-470.

Muller, M., Lerner, H.-W. \& Bolte, M. (2007). Private communication (deposition number 661061). CCDC, Cambridge, England.

Nancy Ghosh, S., Singh, N., Nanda, G. K., Venugopalan, P., Bharatam, P. V. \& Trehan, S. (2003). Chem. Commun. pp. 1420-1421.

Ohwada, T., Achiwa, T., Okamoto, I., Shudo, K. \& Yamaguchi, K. (1998). Tetrahedron Lett. 39, 865-868.

Otani, Y., Nagae, O., Naruse, Y., Inagaki, S., Ohno, M., Yamaguchi, K., Yamamoto, G., Uchiyama, M. \& Ohwada, T. (2003). J. Am. Chem. Soc. 125, 15191-15199.

Pollini, G. P., Benetti, S., De Risi, C. \& Zanirato, V. (2006). Chem. Rev. 106, 2434-2454. 
Sheldrick, G. M. (2015). Acta Cryst. C71, 3-8.

Spande, T. F., Garraffo, H. M., Edwards, M. W., Yeh, H. J. C., Pannell, L. \& Daly, J. W. (1992). J. Am. Chem. Soc. 114, 3475-3478.

Spek, A. L. (2009). Acta Cryst. D65, 148-155.

Sullivan, J. F. \& Bannon, A. W. (1996). CNS Drug Rev. 2, 21-39.
Wang, J., Ma, C., Wu, Y., Lamb, R. A., Pinto, L. H. \& DeGrado, W. F. (2011). J. Am. Chem. Soc. 133, 13844-13847.

Westrip, S. P. (2010). J. Appl. Cryst. 43, 920-925.

Yogeeswari, P., Sriram, D., Bal, T. R. \& Thirumurugan, R. (2006). Nat. Prod. Res. 20, 497-505. 


\section{supporting information}

Acta Cryst. (2017). E73, 1385-1388 [https://doi.org/10.1107/S2056989017012105]

\section{The 7-azanorbornane nucleus of epibatidine: 7-azabicyclo[2.2.1] heptan-7-ium chloride}

\section{Sergey N. Britvin and Andrey M. Rumyantsev}

Computing details

Data collection: APEX2 (Bruker, 2015); cell refinement: SAINT (Bruker, 2015); program(s) used to solve structure: SHELXT (Sheldrick, 2015a) and OLEX2 (Dolomanov et al., 2009); program(s) used to refine structure: SHELXL2014 (Sheldrick, 2015b) and OLEX2 (Dolomanov et al., 2009); molecular graphics: Mercury (Macrae et al., 2008); software used to prepare material for publication: publCIF (Westrip, 2010).

7-Azabicyclo[2.2.1] heptan-7-ium chloride

\section{Crystal data}

$\mathrm{C}_{6} \mathrm{H}_{12} \mathrm{~N}^{+} \cdot \mathrm{Cl}^{-}$

$M_{r}=133.62$

Orthorhombic, $C m c 2_{1}$

$a=9.1532(6) \AA$

$b=8.7029(8) \AA$

$c=8.7336(5) \AA$

$V=695.71(9) \AA^{3}$

$Z=4$

$F(000)=288$

\section{Data collection}

Bruker APEX-II CCD diffractometer

Radiation source: fine focus sealed tube Graphite monochromator $\varphi$ and $\omega$ scans

Absorption correction: multi-scan

(SADABS; Sheldrick, 2015)

\section{Refinement}

Refinement on $F^{2}$

Least-squares matrix: full

$R\left[F^{2}>2 \sigma\left(F^{2}\right)\right]=0.017$

$w R\left(F^{2}\right)=0.048$

$S=1.15$

777 reflections

47 parameters

1 restraint

Hydrogen site location: mixed
$D_{\mathrm{x}}=1.276 \mathrm{Mg} \mathrm{m}^{-3}$

Mo $K \alpha$ radiation, $\lambda=0.71073 \AA$

Cell parameters from 2988 reflections

$\theta=3.2-30.7^{\circ}$

$\mu=0.45 \mathrm{~mm}^{-1}$

$T=100 \mathrm{~K}$

Block, colourless

$0.08 \times 0.06 \times 0.04 \mathrm{~mm}$

3239 measured reflections

777 independent reflections

769 reflections with $I>2 \sigma(I)$

$R_{\text {int }}=0.017$

$\theta_{\max }=27.0^{\circ}, \theta_{\min }=3.2^{\circ}$

$h=-11 \rightarrow 11$

$k=-4 \rightarrow 11$

$l=-11 \rightarrow 10$

$\mathrm{H}$ atoms treated by a mixture of independent and constrained refinement

$w=1 /\left[\sigma^{2}\left(F_{\mathrm{o}}^{2}\right)+(0.0282 P)^{2}+0.1322 P\right]$

where $P=\left(F_{\mathrm{o}}^{2}+2 F_{\mathrm{c}}^{2}\right) / 3$

$(\Delta / \sigma)_{\max }<0.001$

$\Delta \rho_{\max }=0.21 \mathrm{e} \AA^{-3}$

$\Delta \rho_{\min }=-0.12$ e $\AA^{-3}$

Absolute structure: Refined as an inversion twin

Absolute structure parameter: 0.19 (9) 


\section{Special details}

Geometry. All esds (except the esd in the dihedral angle between two 1.s. planes) are estimated using the full covariance matrix. The cell esds are taken into account individually in the estimation of esds in distances, angles and torsion angles; correlations between esds in cell parameters are only used when they are defined by crystal symmetry. An approximate (isotropic) treatment of cell esds is used for estimating esds involving l.s. planes.

Refinement. Single-crystal data collection was performed using a Bruker Kappa APEX II DUO diffractometer equipped with microfocus optics. Refinement of lattice parameters and subsequent data reduction was carried out with the Bruker SAINT software. The crystal structure of 1 was solved and refined using SHELXT and SHELXL-2014 (Sheldrick, 2015) via the OLEX2 v.1.2 graphical user interface (Dolomanov et al., 2009).

Refined as a 2-component inversion twin.

Fractional atomic coordinates and isotropic or equivalent isotropic displacement parameters $\left(\AA^{2}\right)$

\begin{tabular}{lllll}
\hline & $x$ & $y$ & $z$ & $U_{\text {iso }} * / U_{\text {eq }}$ \\
\hline C1 & $0.62232(18)$ & $0.2599(3)$ & $0.5813(2)$ & $0.0174(4)$ \\
H1 & 0.7205 & 0.2960 & 0.5547 & $0.021^{*}$ \\
C2 & $0.5850(2)$ & $0.2715(3)$ & $0.75142(19)$ & $0.0202(4)$ \\
H2B & 0.6233 & 0.3655 & 0.7956 & $0.024^{*}$ \\
H2A & 0.6233 & 0.1843 & 0.8078 & $0.024^{*}$ \\
C6 & $0.5848(2)$ & $0.10083(19)$ & $0.5202(2)$ & $0.0209(4)$ \\
H6A & 0.6231 & 0.0210 & 0.5864 & $0.025^{*}$ \\
H6B & 0.6231 & 0.0863 & 0.4176 & $0.025^{*}$ \\
N7 & 0.5000 & $0.3539(2)$ & $0.5134(2)$ & $0.0139(4)$ \\
H7B & 0.5000 & $0.449(4)$ & $0.548(3)$ & $0.014(7)^{*}$ \\
H7A & 0.5000 & $0.347(4)$ & $0.414(5)$ & $0.030(9)^{*}$ \\
C11 & 0.5000 & $0.31735(5)$ & $0.15788(7)$ & $0.01568(15)$ \\
\hline
\end{tabular}

Atomic displacement parameters $\left(\AA^{2}\right)$

\begin{tabular}{lllllll}
\hline & $U^{11}$ & $U^{22}$ & $U^{33}$ & $U^{12}$ & $U^{13}$ & $U^{23}$ \\
\hline C1 & $0.0107(7)$ & $0.0188(10)$ & $0.0226(9)$ & $0.0012(7)$ & $-0.0009(6)$ & $0.0003(7)$ \\
C2 & $0.0227(10)$ & $0.0240(10)$ & $0.0140(8)$ & $0.0018(8)$ & $-0.0063(7)$ & $0.0029(7)$ \\
C6 & $0.0239(9)$ & $0.0157(9)$ & $0.0232(9)$ & $0.0046(6)$ & $-0.0001(7)$ & $-0.0022(8)$ \\
N7 & $0.0199(10)$ & $0.0119(9)$ & $0.0100(9)$ & 0.000 & 0.000 & $0.0007(8)$ \\
C11 & $0.0213(2)$ & $0.0142(2)$ & $0.0116(2)$ & 0.000 & 0.000 & $0.0005(2)$ \\
\hline
\end{tabular}

Geometric parameters $\left(\stackrel{A}{\circ}{ }^{\circ}\right)$

\begin{tabular}{llll}
\hline $\mathrm{C} 1-\mathrm{H} 1$ & 0.9800 & $\mathrm{C} 6-\mathrm{C}^{\mathrm{i}}$ & $1.553(4)$ \\
$\mathrm{C} 1-\mathrm{C} 2$ & $1.528(2)$ & $\mathrm{C} 6-\mathrm{H} 6 \mathrm{~A}$ & 0.9700 \\
$\mathrm{C} 1-\mathrm{C} 6$ & $1.523(3)$ & $\mathrm{C} 6-\mathrm{H} 6 \mathrm{~B}$ & 0.9700 \\
$\mathrm{C} 1-\mathrm{N} 7$ & $1.508(2)$ & $\mathrm{N} 7-\mathrm{C}^{\mathrm{i}}$ & $1.508(2)$ \\
$\mathrm{C} 2-\mathrm{C} 2^{\mathrm{i}}$ & $1.556(4)$ & $\mathrm{N} 7-\mathrm{H} 7 \mathrm{~B}$ & $0.88(3)$ \\
$\mathrm{C} 2-\mathrm{H} 2 \mathrm{~B}$ & 0.9700 & $\mathrm{~N} 7-\mathrm{H} 7 \mathrm{~A}$ & $0.87(4)$ \\
$\mathrm{C} 2-\mathrm{H} 2 \mathrm{~A}$ & 0.9700 & & $103.03(9)$ \\
& & & 111.2 \\
$\mathrm{C} 2-\mathrm{C} 1-\mathrm{H} 1$ & 114.5 & $\mathrm{C} 1-\mathrm{C} 6-\mathrm{C} 6{ }^{\mathrm{i}}$ & 111.2 \\
$\mathrm{C} 6-\mathrm{C} 1-\mathrm{H} 1$ & 114.5 & $\mathrm{C} 1-\mathrm{C} 6-\mathrm{H} 6 \mathrm{~A}$ & \\
$\mathrm{C} 6-\mathrm{C} 1-\mathrm{C} 2$ & $110.50(19)$ & $\mathrm{C} 1-\mathrm{C} 6-\mathrm{H} 6 \mathrm{~B}$ &
\end{tabular}




\begin{tabular}{|c|c|c|c|c|}
\hline $\mathrm{N} 7-\mathrm{C} 1-\mathrm{H} 1$ & 114.5 & $\mathrm{C} 6-\mathrm{C} 6-\mathrm{H} 6 \mathrm{~A}$ & & 111.2 \\
\hline $\mathrm{N} 7-\mathrm{C} 1-\mathrm{C} 2$ & $100.39(16)$ & $\mathrm{C} 6-\mathrm{C} 6-\mathrm{H} 6 \mathrm{~B}$ & & 111.2 \\
\hline $\mathrm{N} 7-\mathrm{C} 1-\mathrm{C} 6$ & $100.82(15)$ & $\mathrm{H} 6 \mathrm{~A}-\mathrm{C} 6-\mathrm{H} 6 \mathrm{~B}$ & & 109.1 \\
\hline $\mathrm{C} 1-\mathrm{C} 2-\mathrm{C} 2^{\mathrm{i}}$ & $102.93(9)$ & $\mathrm{C} 1-\mathrm{N} 7-\mathrm{C}^{\mathrm{i}}$ & & $95.91(18)$ \\
\hline $\mathrm{C} 1-\mathrm{C} 2-\mathrm{H} 2 \mathrm{~B}$ & 111.2 & $\mathrm{C} 1-\mathrm{N} 7-\mathrm{H} 7 \mathrm{~B}$ & & $111.8(11)$ \\
\hline $\mathrm{C} 1-\mathrm{C} 2-\mathrm{H} 2 \mathrm{~A}$ & 111.2 & $\mathrm{C} 11^{\mathrm{i}}-\mathrm{N} 7-\mathrm{H} 7 \mathrm{~B}$ & & $111.8(11)$ \\
\hline $\mathrm{C} 2-\mathrm{C} 2-\mathrm{H} 2 \mathrm{~B}$ & 111.2 & $\mathrm{C} 1-\mathrm{N} 7-\mathrm{H} 7 \mathrm{~A}$ & & $110.6(14)$ \\
\hline $\mathrm{C} 2{ }^{\mathrm{i}}-\mathrm{C} 2-\mathrm{H} 2 \mathrm{~A}$ & 111.2 & $\mathrm{C} 11^{\mathrm{i}}-\mathrm{N} 7-\mathrm{H} 7 \mathrm{~A}$ & & $110.6(14)$ \\
\hline $\mathrm{H} 2 \mathrm{~B}-\mathrm{C} 2-\mathrm{H} 2 \mathrm{~A}$ & 109.1 & $\mathrm{H} 7 \mathrm{~B}-\mathrm{N} 7-\mathrm{H} 7 \mathrm{~A}$ & & $115(3)$ \\
\hline $\mathrm{C} 2-\mathrm{C} 1-\mathrm{C} 6-\mathrm{C}^{\mathrm{i}}$ & $70.63(12)$ & $\mathrm{C} 6-\mathrm{C} 1-\mathrm{N} 7-\mathrm{C} 1^{\mathrm{i}}$ & & $56.31(19)$ \\
\hline $\mathrm{C} 2-\mathrm{C} 1-\mathrm{N} 7-\mathrm{C}^{\mathrm{i}}$ & $-57.1(2)$ & $\mathrm{N} 7-\mathrm{C} 1-\mathrm{C} 2-\mathrm{C} 2^{\mathrm{i}}$ & & $35.24(15)$ \\
\hline $\mathrm{C} 6-\mathrm{C} 1-\mathrm{C} 2-\mathrm{C}^{\mathrm{i}}$ & $-70.56(14)$ & $\mathrm{N} 7-\mathrm{C} 1-\mathrm{C} 6-\mathrm{C} 6^{\mathrm{i}}$ & & $-34.89(12)$ \\
\hline \multicolumn{5}{|c|}{ Symmetry code: (i) $-x+1, y, z$. } \\
\hline \multicolumn{5}{|c|}{ Hydrogen-bond geometry $\left(\AA,{ }^{o}\right)$} \\
\hline$D-\mathrm{H} \cdots A$ & $D-\mathrm{H}$ & $\mathrm{H} \cdots A$ & $D \cdots A$ & $D-\mathrm{H} \cdots A$ \\
\hline $\mathrm{N} 7-\mathrm{H} 7 B \cdots \mathrm{Cl} 1^{\mathrm{ii}}$ & $0.88(3)$ & $2.25(3)$ & $3.127(2)$ & $175(2)$ \\
\hline $\mathrm{N} 7-\mathrm{H} 7 A \cdots \mathrm{Cl} 1$ & $0.87(4)$ & $2.25(4)$ & $3.122(2)$ & $178(3)$ \\
\hline
\end{tabular}

Symmetry code: (ii) $-x+1,-y+1, z+1 / 2$. 